\title{
Unlearning as a function of degree of original learning and retention test ${ }^{1,2}$
}

BERTRAM E. GARSKOF, MICHIGAN STATE UNIVERSITY TOMMIE M. BRYAK, RUTGERS, THE STATE UNIVERSITY

Three degrees of original learning (OL) and two retention tests were used to explore the relationship between degree of original learning and resistance to unlearning. It was found that scores on both retention tests varied directly with degree of $O L$ suggesting that greater $O L$ practice led to increased resistance to unlearning during interpolated learning.

One source of retroactive inhibition (RI) is the unlearning of original learning (OL) during interpolated learning (Barnes \& Underwood, 1959). Recent studies (Postman, Keppel * \& Stark, 1965; Postman, 1965) suggest that unlearning increases with the frequency with which OL responses are elicited during interpolated learning (IL). The present study explores the notion that increased degree of OL will render OL items more resistant to unlearning during their elicitation in IL.

McGovern (1964) has indicated that unlearning is a 2stage process consisting of response unlearning and $S-R$ or associative unlearning. Modified Modified Free Recall (MMFR), which demands response recall may be primarily sensitive to response unlearning while another retention test, S-R Matching in which Ss are given the responses and asked only to recognize the proper $S-R$ pairings is primarily a measure of S-R unlearning. Both tests were employed in the present study to determine the effect of degree of OL on the course of the two components of unlearning.

\section{Method}

Three degrees of first list learning and the two retention measures were used in a 3 by 2 factorial design. Ss learned a list of eight paired associates (A-B) to a criterion of correct anticipation of four responses out of the eight pairs $(E-1)$, or to a criterion of one correct anticipation (E-2) or six correct anticipations (E-3) of the list. All experimental Ss were then presented with a second list $(\mathrm{A}-\mathrm{C})$ for 10 trials. Two control groups learned $A-B$ to $E-1$ 's criterion and then either received 10 trials on a D-C list $(C-1)$ or engaged in an irrelevant design judgment task (Graves, 1946) for a period of time equal to that required for the $10 \mathrm{IL}$ trials (C-2). Immediately after IL, half the Ss in each condition were tested with the MMFR measure of retention, while the other half were tested with the S-R Matching measure.

The two paired-associate lists employed by Barnes \& Underwood (1959) in the A-B, A-C paradigm were used in the present experiment. Stimuli were eight CVC nonsense syllables; responses were two-syllable adjectives. An additional eight CVC nonsense syllables from the McGovern (1964) study were used as D terms in the A-B, D-C control group. Two of Barnes and Underwood's three different pairings of stimuli and responses were used. An equal number of Ss in each condition were given each pairing. Five orders of items were used to minimize serial learning.

The retention booklets contained eight pages, one for each stimulus word. Each stimulus word was printed once and was followed by a blank space. The pages for each booklet were shuffled before being stapled. An additional sheet listing the responses in a mixed order was available for the S-R Matching test.

Ten Rutgers female undergraduate Ss were assigned randomly to each of the six experimental and four control conditions in the order of their appearance in the laboratory.

Standard paired-associate learning instructions were read to Ss. Lists were presented on a Lafayette memory drum at a 2:2 rate with $4 \mathrm{sec}$. between trials and $1 \mathrm{~min}$. between lists or irrelevant task. The two retention tests were administered immediately after 10 trials on the second list or after an equal amount of time spent on the design judgment task. Ss were asked to write the appropriate list 1 responses in the retention booklets. They were allowed to work at their own pace, but were not permitted to return to a page once it had been turned. In the Matching task, a sheet containing first list responses was presented with the booklet.

\section{Resulis}

The A-B learning data were examined to determine if there was any evidence for group differences in learning ability. The number of trials required to reach the low criterion used in E-1, C-1, and C-2 was tabulated for the E-2 and E-3 groups. An analysis of variance of these data revealed no significant effect of conditions or of retention measures, nor was the interaction significant (Fs $<1$ ). A similar analysis of variance of the number of trials required by the E-2 and E-3 groups to reach the criterion of one perfect anticipation disclosed no significant effect of experimental conditions or of retention measures and no significant interaction (Fs $<1$ ). A " $t$ " test between the number of trials to the criterion of six perfect anticipations for the two retention groups in E-3 was not significant $(t=.23)$. Thus, no analysis revealed significant differences between groups on first list learning.

The mean number of correct pairings for each condition on the MMFR and Matching tests are presented in Table 1 . The number of correct pairings during recall for each $\mathbf{S}$ was converted to a proportion and an arcsine transformation employed to obtain normality of distribution and homogeneity of variance. 
Table 1. Means and Standard Deviations for Number of Correctly Recalled or Paired Items

\begin{tabular}{llll} 
& & MMFR & Matching \\
\hline E-1 & Mean & 1.8 & 3.5 \\
& S.D. & 1.33 & 1.02 \\
E-2 & Mean & 3.8 & 5.4 \\
& S.D. & 1.60 & 1.80 \\
E-3 & Mean & 6.1 & 7.7 \\
& S.D. & .94 & .45 \\
C-1 & Mean & 3.7 & 4.5 \\
& S.D. & 2.24 & 1.57 \\
C-2 & Mean & 5.8 & 6.0 \\
& S.D. & 1.40 & 1.90 \\
\hline
\end{tabular}

An analysis of variance of the transformed data from the three experimental groups revealed a significant effect of the retention measure employed $(F=28.34, \mathrm{df}=$ $1 / 54, p<.01$ ) and of the degree of first list learning ( $F=$ 51.36, $\mathrm{df}=2 / 54, \mathrm{p}<.01$ ). The interaction between measures and experiment conditions was non-significant $(F<1)$. The Newman-Keuls procedure indicated that the three experimental groups differed significantly $(p<.05)$ on both measures and the two retention measures differed significantly $(p<.05)$ at each degree of learning.

The E-1 condition was compared with two control groups to determine if there was direct evidence for unlearning at this degree of $O L$. An analysis of variance for $\mathrm{E}-1, \mathrm{C}-1$, and $\mathrm{C}-2$ showed a significant effect of the measure employed $(F=4.90, \mathrm{df}=1 / 54, \mathrm{p}<.05)$ and of the interpolated task $(\mathrm{F}=16.95, \mathrm{df}=2 / 54, \mathrm{p}<.01)$. The interaction between measures and interpolated learning conditions was non-significant $(F<1)$. On the MMFR measure, the Newman-Keuls procedure indicated that the means of the three groups differed significantly from each other $(p<.05)$. Comparisons between groups on the Matching test indicated that E-1 and C-2 differed from each other $(p<.01)$, but that neither differed from $C-1$ $(p<.05)$. Scores on the MMFR and Matching tests differed significantly only in the $E-1$ condition ( $F=5.28$, $\mathrm{df}=1 / 54, \mathrm{p}<.05$ ).

\section{Discussion}

OL retention increased on both tests as the amount of OL practice increased. There was no evidence for an interaction between degree of OL and type of test. Where direct comparision between experimental and control groups was possible (E-1 vs. $\mathrm{C}-1$ and $\mathrm{C}-2$ ) it was clear that the interpolation of a second learning list led to RI even at this low degree of OL. Since retention tests employed minimize response competition the differences between work and control groups may be presumed to reflect the unlearning of OL items.

Indirect evidence can be adduced for the conclusion that unlearning occurred as well in the E-2 and E-3 groups in MMFR and in the E-2 group on the Matching test. The control group for normal forgetting used in
Barnes \& Underwood (1959) practiced the A-B listuntil a criterion of one perfect repetition of the list was attained. After an irrelevant task mean number of items correctly recalled and paired in MMFR was 7.75. It would appear safe to infer that a $\mathrm{C}-2$ group run to the same OL criterion as the E-3 group in the present study would show virtually perfect recall. Thus, MMFR performance in E-2 and E-3 was considerably lower than that which would occur in comparable control groups. The same argument can also be made for the E-2 group in the matching condition where performance was lower that what the comparable control would probably show. In E-3, however, matching was almost errorless.

The present data seem to permit the conclusions that response unlearning occurred at all levels of OL tested and that associative unlearning occurred at all but the highest level of OL where the additional practice evidently mitigates the effect of IL. These data then suggest that degree of OL practice relates directly to the extent to which OL items can resist the extinctive effects of IL. The lack of interaction between the two tests suggest that the processes they are measuring, if different, are not differentially affected by OL practice.

The question of associative unlearning in the $A-B, D-C$ paradigm can be examined in these data. Current theory of unlearning and some evidence (McGovern, 1965) indicate that no decrement should be found in the A-B, D-C paradigm when a matching test is used. However, Garskof (1964) has previously reported decrements in this situation. This decrement is again found here where the performance of the $\mathrm{C}-1$ group is significantly lower than the $\mathrm{C}-2$ group on the matching test. Further research is needed to settle this issue.

\section{References}

Bames, J. M., \& Underwood, B. J. "Fate" of first list associations in transfer theory. J. exp. Psychol., 1959, 58, 97-105.

Garskof, B. E. Unlearning in recognition matching as a function of degree of second iist learning. Paper presented at Psychonomic Society meeting, 1964 .

Graves, M. Design judgement test. New York: Psychological Corp., 1946.

McGovem, J. B. Extinction of associations in four transfer paradigms. Psychol. Monogr., 1964, 78, No. 16. Whole No. 593.

Postman, L. Unlearning under conditions of successive interpolation. J. exp. Psychol., 1965, 70, 237-245.

Postman, L., Keppel, G., \& Stark, K. Unlearning as a function of the relationship between successive response classes. J. exp. Psychol., 1965, 69, 111-118.

\section{Notes}

1. This research was supported in part by a grant from the Research Council of Rutgers University and by grant number 02626 from the National Institute of Child Health and Human Development to the senior author.

2. This report is based upon a thesis submitted by the junior author to Rutgers, The State University in partial fulfillment of the requirements for the M.A. degree. 